\title{
Electronic Dispersion Compensation of 40-Gb/s Multimode Fiber Links Using IIR Equalization
}

\author{
G. Ng and A. Chan Carusone \\ Edward S. Rogers Dept. of Electrical \& Computer Engineering, University of Toronto, \\ 10 King's College Rd. Toronto, ON, M5S 3G4, Canada \\ ngg@eecg.toronto.edu \\ tcc@eecg.toronto.edu
}

\begin{abstract}
Simulations demonstrate a 2nd order IIR equalizer compensates for modal dispersion in over $90 \%$ of the $5 \%$ worst-case $50-\mathrm{m}$ multimode fiber links at $40 \mathrm{Gbps}$ with less than $2.5 \mathrm{~dB}$ ISI penalty and 0.15 UI of jitter.

(C) 2006 Optical Society of America

OCIS codes: (060.2330) Fiber optics communications (060.2360) Fiber optics links and subsystems
\end{abstract}

\section{Introduction}

Recently there has been increased interest in using multimode fiber for data transmission at speeds greater than 10 $\mathrm{Gb} / \mathrm{s}$. Multimode fiber (MMF) is attractive for short haul multi-gigabit communications due to its high bandwidth compared to coaxial links.

The major limiting factor in data transmission over short multimode fiber links is intermodal dispersion. Intermodal dispersion is caused when different fiber mode groups are excited, each having a different propagation velocity. This causes inter-symbol interference (ISI) at the receiver. Although it has been demonstrated that intermodal dispersion can be compensated in the optoelectronic domain [1], the most robust and cost-effective method of mitigating such ISI is by electronic equalization. Traditionally, electronic equalization of $10-\mathrm{Gb} / \mathrm{s} \mathrm{MMF}$ links involved using finite impulse response (FIR) equalizers (Fig. 1a), or decision feedback equalizers (DFE) (Fig. 1b). This paper investigates the transmission and equalization of a $40-\mathrm{Gb} / \mathrm{s}$ data stream over $50 \mathrm{~m}$ of MMF using an infinite impulse response (IIR) filter.

\section{IIR Equalizer}

A basic direct-form $2^{\text {nd }}$ order IIR filter is shown in Fig. 1c. Coefficients $b_{0}, b_{1}$, and $b_{2}$ are in the feed-forward path, coefficients $a_{1}$ and $a_{2}$ are in the feedback path, and $\tau$ is the delay between taps. By changing the coefficients $b_{i}$ and $a_{i}$, the positions of the zeros and poles, respectively, can be manipulated. This is unlike an FIR equalizer whose pole locations are fixed. With the proper selection of the poles and zeros, an IIR equalizer can provide more flexibility in the shape of the frequency response than a FIR equalizer. In the time domain, the IIR equalizer's impulse response can be thought of as creating additional post-cursor cancelling taps beyond the reach of the FIR equalizer. This means that an IIR equalizer with the same span as an FIR equalizer can potentially cancel ISI beyond the reach of the FIR equalizer. Unlike the DFE, the IIR equalizer is linear and operates in continuous time. So whereas a DFE must consume a lot of power to meet the timing requirements of its critical path, especially at $40 \mathrm{~Gb} / \mathrm{s}$ [2], in an IIR equalizer the timing requirements are relaxed. Timing recovery is also simplified using the IIR equalizer instead of the DFE.

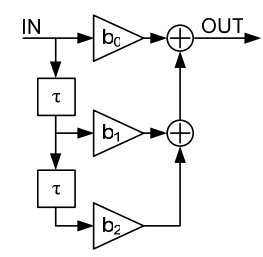

(a)

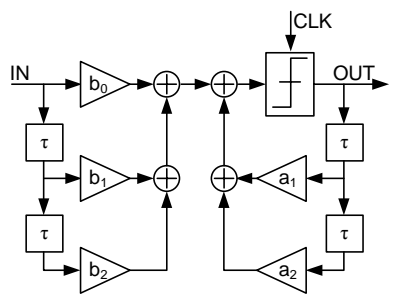

(b)

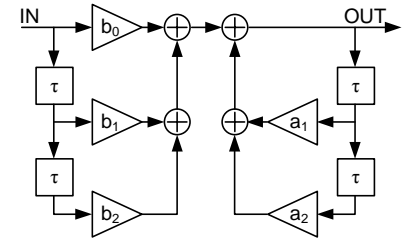

(c) Fig. 1 Electronic equalizer structures. (a) 3-tap FIR equalizer. (b) DFE. (c) $2^{\text {nd }}$ order IIR filter.

\section{Fiber Channel System Simulation}

To verify the effectiveness of using an IIR equalizer for ISI cancellation, a statistical multimode fiber model created by the 802.3aq task force was used [3]. This "108-fiber model" of FDDI-grade MMF represents the performance of 
the worst-case $5 \%$ of fibers that are likely to be installed [4]. The modeled fibers have a core diameter of $62.5 \mu \mathrm{m}$ and a laser source wavelength of $1300 \mathrm{~nm}$. Various defects in the refractive-index profiles in legacy multimode fibers are modeled. All fiber models have a maximum differential delay target of $2 \mathrm{~ns} / \mathrm{km}$ and an overfilled-launch bandwidth-distance of $500 \mathrm{MHz}-\mathrm{km}$. The model is based on a restricted launch scheme where the light signal is coupled into the fiber either from a lens or a single-mode fiber. A total of 108 different fibers at 17, 20 and $23 \mu \mathrm{m}$ radial offset launch positions were studied. The average modal delays were linearly scaled from the $300 \mathrm{~m}$ fibers in the original model down to $50 \mathrm{~m}$.

The model of the MMF channel and equalizer system used in simulations is shown in Fig. 2. Assuming the operating wavelength is near the fiber's zero dispersion wavelength, the effects of chromatic dispersion are minimal. Hence, only intermodal dispersion was modeled in the "MMF Fiber Channel" block. A transimpedance amplifier (TIA) and variable gain amplifier (VGA) ensure that the amplitude of the signal at the input of the equalizer is fullscale with zero DC offset. Hence, an offset-free 40-Gb/s NRZ bipolar signal was used instead of an on-off bit source. The transmitter and receiver front-ends are modeled by first order low-pass filters with a bandwidth of $0.8 \times$ bit-rate. Either a 3-tap FIR or a $2^{\text {nd }}$ order IIR filter was used for channel equalization.

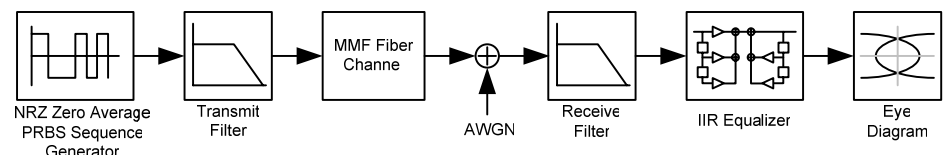

Fig. 2 MMF fiber link model.

ISI penalty at the equalizer output and data-dependent peak-to-peak jitter were used as the figures of merit. ISI penalty is defined as $10 \log _{10}\left(\mathrm{~A}_{0} / \mathrm{A}_{\mathrm{n}}\right)$, where $\mathrm{A}_{0}$ is the difference between the upper and lower lines in the eye diagram (the DC response of the channel) and $A_{n}$ is the maximum vertical eye opening considering only ISI [5]. Hence, when the eye is completely open, the ISI penalty is zero, while a closed eye has infinite ISI penalty.

The $2^{\text {nd }}$ order IIR equalizer coefficients were optimized iteratively. First, a long minimum mean square error (MMSE) FIR equalizer response was calculated. Then, a nonlinear least squares function using the LevenbergMarquardt method with line search was used to find IIR coefficients $b_{1}, b_{2}, b_{3}, a_{1}$ and $a_{2}$ that produce an impulse response that best match the MMSE FIR impulse response. The number of taps in the MMSE FIR equalizer was then increased and the process was repeated until the nonlinear least squares function failed to find an IIR filter that matches the long FIR impulse response.

\section{Simulated IIR Equalizer Performance Results}

The performance of a $2^{\text {nd }}$ order fractionally spaced IIR equalizer was compared to the performance of a 3-tap fractionally spaced FIR equalizer. Both equalizers have a tap spacing of $\tau=12.5 \mathrm{ps}$ and a total feed-forward delay of $2 \tau=25 \mathrm{ps}$. The input $40-\mathrm{Gb} / \mathrm{s}$ bit source has a period of $25 \mathrm{ps}$.

Fig. 3 illustrates the effectiveness of using an IIR equalizer with an example difficult channel from the $17 \mu \mathrm{m}$ offset launch data set. In this particular channel the pulse response full-width half-max spans slightly more than 2 bit periods (54.8 ps), thus the eye is closed as illustrated in Fig. 3a. The ISI penalty of the unequalized eye is $20.3 \mathrm{~dB}$ and the data-dependent peak-to-peak jitter is $21.4 \mathrm{ps}$. Equalizing the channel with a 3-tap FIR filter results in the partially open eye shown in Fig. 3b. The ISI penalty has been reduced to $3.9 \mathrm{~dB}$ and the data-dependent peak-topeak jitter is 11.4 ps. Using a $2^{\text {nd }}$ order IIR equalizer results in a completely open eye. The resulting equalized eye is shown in Fig. 3c. The ISI penalty is only $1.1 \mathrm{~dB}$ and the data-dependent peak-to-peak jitter is 3.3 ps which is significantly better than that of the FIR filter.

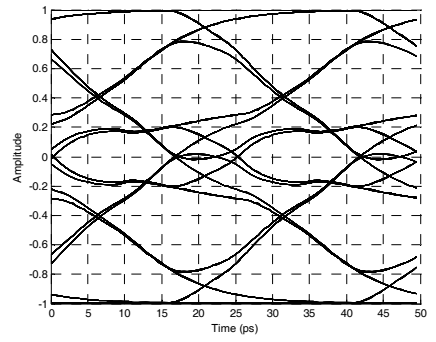

(a)

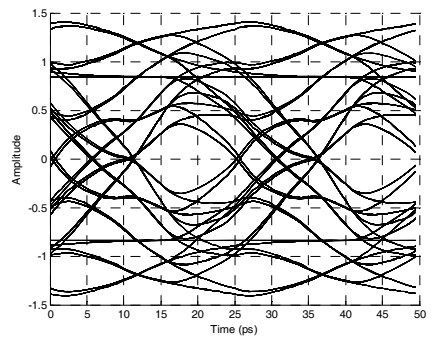

(b)

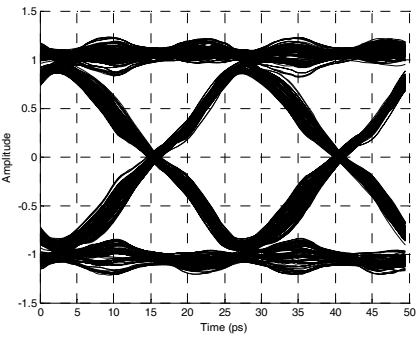

(c)

Fig. 3 An example fiber with offset launch of $17 \mu \mathrm{m}$ showing the effectiveness of using and IIR equalizer. (a) Channel output eye before equalization. (b) 3 -tap FIR equalized eye. (c) $2^{\text {nd }}$ order IIR equalized eye. 
A statistical analysis was performed to verify that using the IIR equalizer has a significant benefit over the FIR equalizer. All 108 fiber channels at the three different offset launch positions were equalized with the FIR and IIR equalizers. The equalized ISI penalty and data-dependent peak-to-peak jitter histograms for all three offset launches shown in Fig. 4, clearly demonstrate that there is an overall improvement in eye quality when using the IIR equalizer. For offset launches of 17, 20 and $23 \mu \mathrm{m}$, the IIR equalizer had better performance than the FIR equalizer in $78.7 \%$, $80.6 \%$ and $74.1 \%$ of the fibers respectively. The IIR equalizer never has worse ISI penalty performance than the FIR equalizer since the IIR equalizer can be used as a 3-tap FIR filter with the feedback coefficients $a_{1}$ and $a_{2}$ set to zero. Targeting a maximum ISI penalty of $2.5 \mathrm{~dB}$ and a maximum pattern-dependent peak-to-peak jitter of less than $15 \%$ of the bit period $(0.15 \mathrm{UI}=3.75 \mathrm{ps})$, the $2^{\text {nd }}$ order IIR equalizer works for $91.7 \%, 94.4 \%$ and $90.7 \%$ of all the $5 \%$ worst-case $50 \mathrm{~m}$ MMF channels at $40 \mathrm{~Gb} / \mathrm{s}$ at offset launches of 17,20 and $23 \mu \mathrm{m}$ respectively. A summary of the results are tabulated in Table 1.
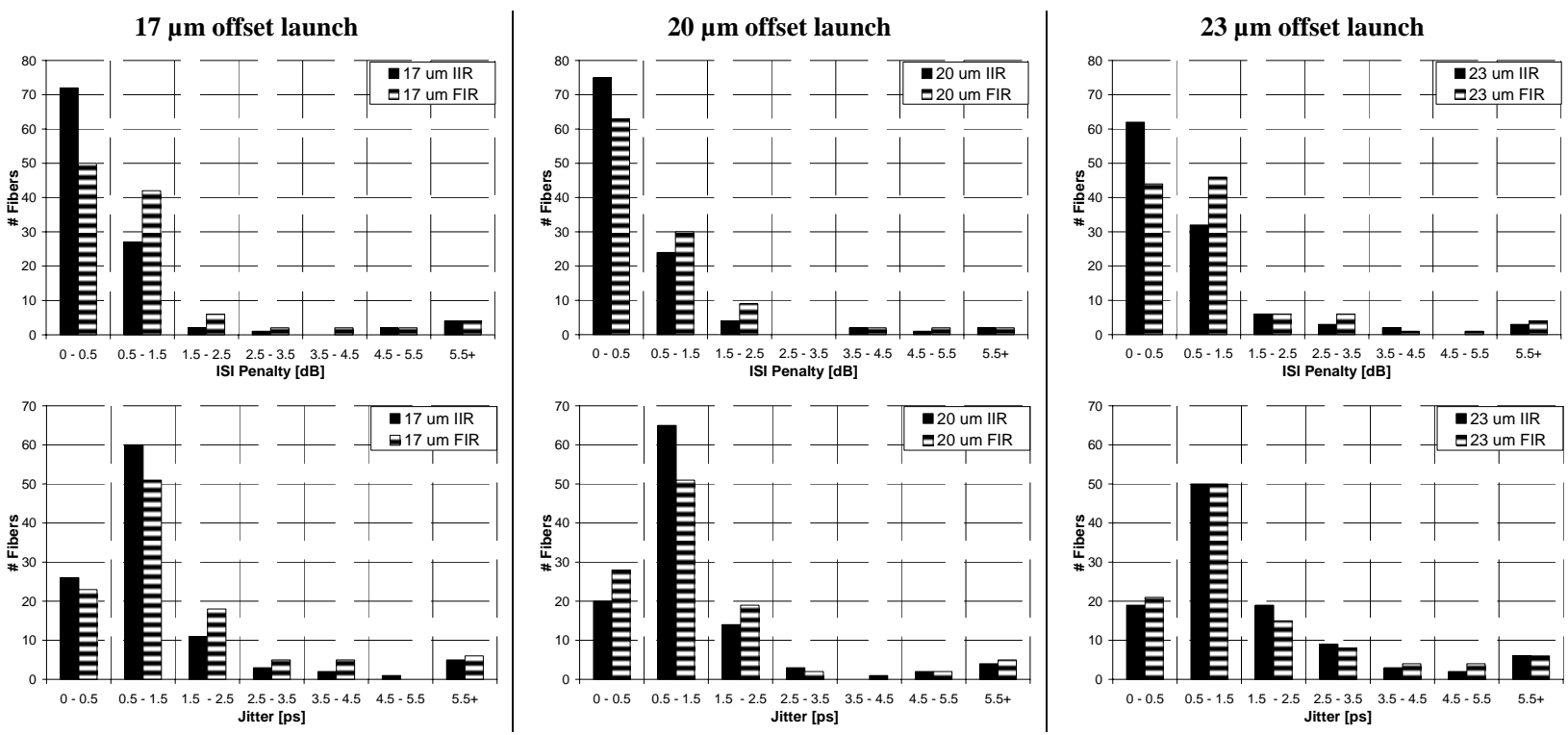

Fig. 4 Histograms showing the improvement in both ISI penalty (top row) and data-dependent peak-to-peak jitter (bottom row) when comparing the IIR (solid black) with the FIR (striped) equalizer for all three offset launches.

Table 1. Summary of statistical simulation results. $\mathrm{DD}=$ Data-dependent.

\begin{tabular}{|l|l|l|l|l|}
\hline $\begin{array}{l}\text { Launch } \\
\text { Offset }\end{array}$ & $\begin{array}{l}\text { \% of Fibers } \\
\text { Benefiting from Using } \\
\text { IIR Equalization }\end{array}$ & $\begin{array}{l}\text { \% of Equalized Fibers } \\
\text { with less than 2.5 dB } \\
\text { ISI Penalty }\end{array}$ & $\begin{array}{l}\text { \% of Equalized Fibers with } \\
\text { less than 0.15 UI (3.75 ps) of } \\
\text { DD Peak-to-peak Jitter }\end{array}$ & $\begin{array}{l}\text { \% of Equalized Fibers with } \\
<2.5 \text { dB ISI Penalty and } \\
<3.75 \text { ps DD Peak-to-peak Jitter }\end{array}$ \\
\hline $\mathbf{1 7} \mathbf{~} \mathbf{m}$ & $78.7 \%$ & $93.5 \%$ & $93.5 \%$ & $91.7 \%$ \\
\hline $\mathbf{2 0} \mathbf{~ m}$ & $80.6 \%$ & $95.4 \%$ & $94.4 \%$ & $94.4 \%$ \\
\hline $\mathbf{2 3} \mathbf{~} \mathbf{m}$ & $74.1 \%$ & $92.6 \%$ & $91.7 \%$ & $90.7 \%$ \\
\hline
\end{tabular}

\section{Conclusion}

Through system simulations, the effectiveness of using a $2^{\text {nd }}$ order IIR equalizer over a FIR equalizer was demonstrated. The IIR equalizer offers better performance than a 3-tap FIR equalizer more than $74 \%$ of the time, and is capable of equalizing more than $90 \%$ of all the $5 \%$ worst-case $50 \mathrm{~m}$ MMF channels at $40 \mathrm{~Gb} / \mathrm{s}$ with a maximum ISI penalty of $2.5 \mathrm{~dB}$ and less than $3.75 \mathrm{ps}$ of data-dependent peak-to-peak jitter.

\section{References}

[1] K.M. Patel and S.E. Ralph, "Multimode Fiber Link Equalization by Mode Filtering via a Multisegment Photodetector," in Microwave Symposium Digest, 2003 IEEE MTT-S International, vol.2, no.pp. 1343- 1346 vol.2, 8-13 June 2003

[2] A. Garg, A.C. Carusone, and S.P. Voinigescu, "A 1-Tap 40-Gb/s Look-Ahead Decision Feedback Equalizer in 0.18- $\mu$ m SiGe BiCMOS Technology," in Solid-State Circuits, IEEE Journal of, vol.41, no.10pp. 2224- 2232, Oct. 2006

[3] IEEE 802.3aq Task Force: http://www.ieee802.org/3/aq/public/tools/108fiberModel/

[4] M. Webster, L. Raddatz, I.H. White and D.G. Cunningham, "A Statistical Analysis of Conditioned Launch for Gigabit Ethernet Links Using Multimode Fiber," in Lightwave Technology, Journal of, vol.17, no.9pp.1532-1541, Sep 1999

[5] P. Pepeljugoski, S.E. Golowich, A.J. Ritger, P. Kolesar, and A. Risteski, "Modeling and simulation of next-generation multimode fiber links," in Lightwave Technology, Journal of, vol.21, no.5pp. 1242- 1255, May 2003 